\title{
Imaging in Non-Traumatic Emergencies: Acute Thrombosis of the Aorta and Pulmonary Embolism
}

\author{
Bennidor Raviv, Shlomo Hanan Israelit
}

Department of Emergency Medicine, Rambam Health Care Campus, Haifa, Israel

\begin{abstract}
:
Objectives: To present the possibility of acute arterial and venous thrombosis.
\end{abstract}

Materials and methods: Report of a patient presenting with acute dyspnoea and chest pain.

Results: Using a combined medical team and imaging studies, pulmonary embolism and acute arterial thrombosis were diagnosed. The patient was treated medically and surgically.

Conclusion: Physicians should be aware of the possibility of combined thrombosis and the diagnosis and management of the condition.

Keywords: Arterial thrombosis, pulmonary embolism, emergency

Received: $14 / 04 / 2014$

Accepted: $23 / 04 / 2014$

Published: 19/05/2014

How to cite this article: Raviv B, Israelit SH. Imaging in Non-Traumatic Emergencies: Acute Thrombosis of the Aorta and Pulmonary Embolism, EJCRIM 2014;1:doi: 10.12890/2014_000076

Conflicts of Interests: The authors declare that they have no conflicts of interest in this research. 


\section{Case presentation}

A 63-year-old woman presented to the emergency department with 1-hour onset of chest and back pain. She had been discharged from our hospital one week previously after undergoing coronary artery bypass grafting (CABG) surgery.

On admission, the patient was hypotensive and tachycardic and no peripheral pulses were palpated. She complained of a crushing pain in her back and left hand.

After fluid resuscitation, the patient stabilized haemodynamically, but still no peripheral pulses were felt.

A mobile chest X-ray did not demonstrate any pathology that could explain the signs and symptoms; hence an urgent bedside trans-esophageal echocardiogram (TEE) was performed to rule out dissection of the aorta. No dissection was found, but a thrombus was seen at the origin of the left subclavian artery. A decision was made to proceed to biphasic (arterial and venous) computed tomography (CT) scan, because there was clinical suspicion of both arterial and pulmonary venous thrombosis. Due to an iodine allergy and lack of time for preparation, the patient was intubated.

The CT scan demonstrated two different pathologies: thrombi in the main pulmonary arteries, extending into the lobar and segmental branches; and arterial thrombus beginning at the aortic arch, extending to the left subclavian artery and the right axial artery. No thrombi were demonstrated in the carotid arteries (Figs. 1-3).

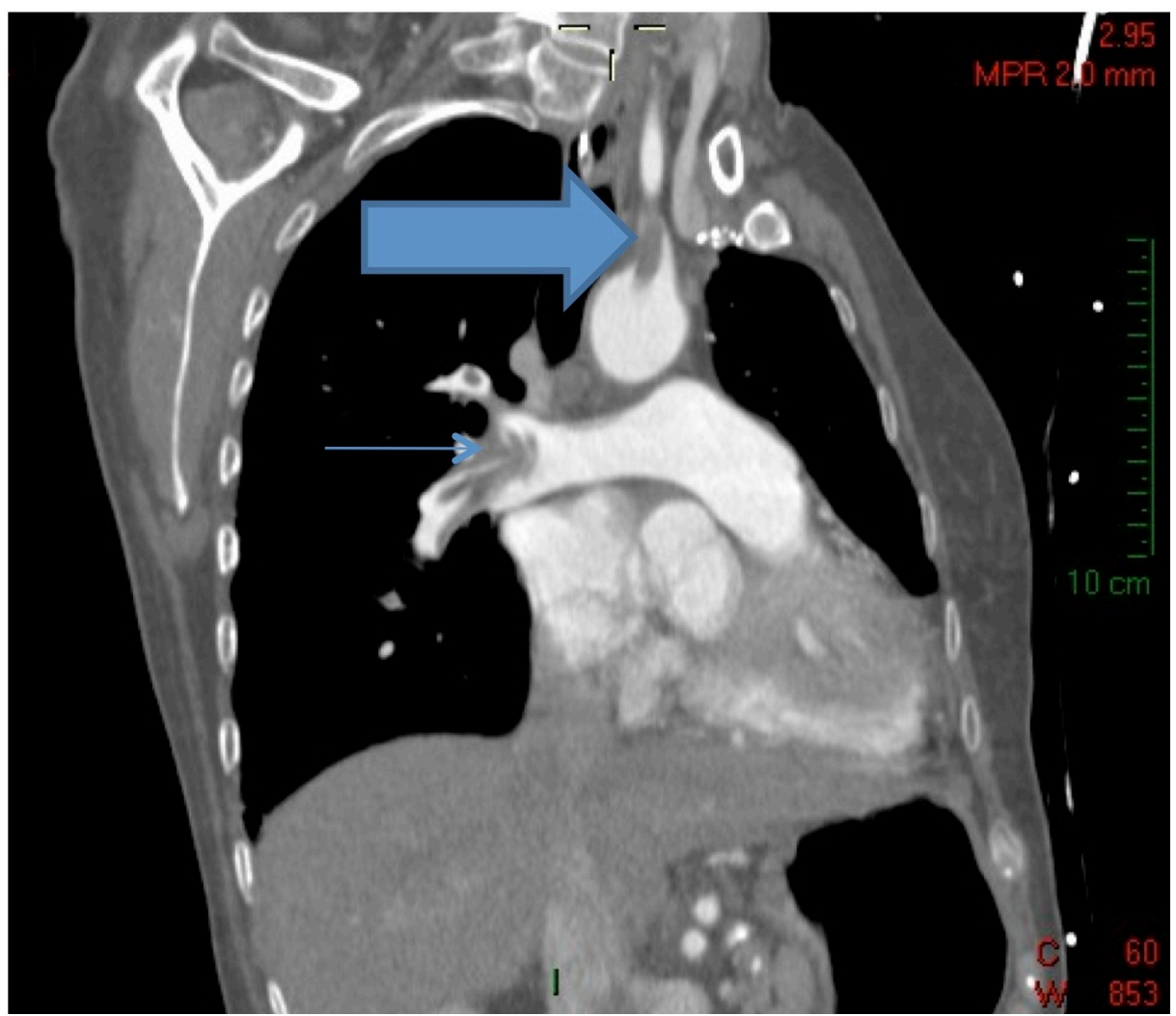

Figure 1: Arterial thrombosis in the ascending aorta and right subclavian artery (thick arrow) and venous thrombosis in the main right pulmonary artery trunk (thin arrow). 


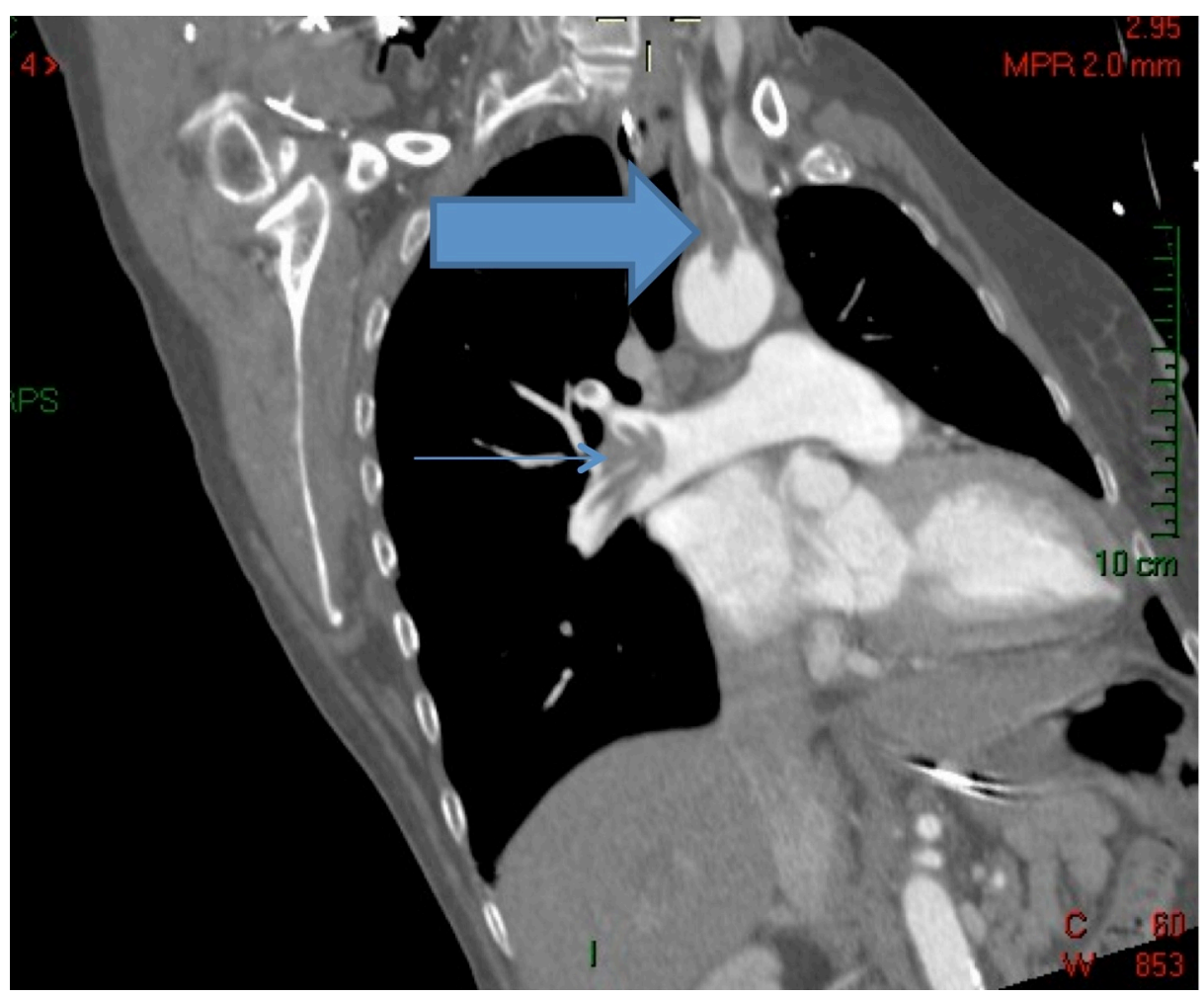

Figure 2: Arterial thrombosis in the ascending aorta and right subclavian artery (thick arrow) and venous thrombosis in the main right pulmonary artery trunk (thin arrow).

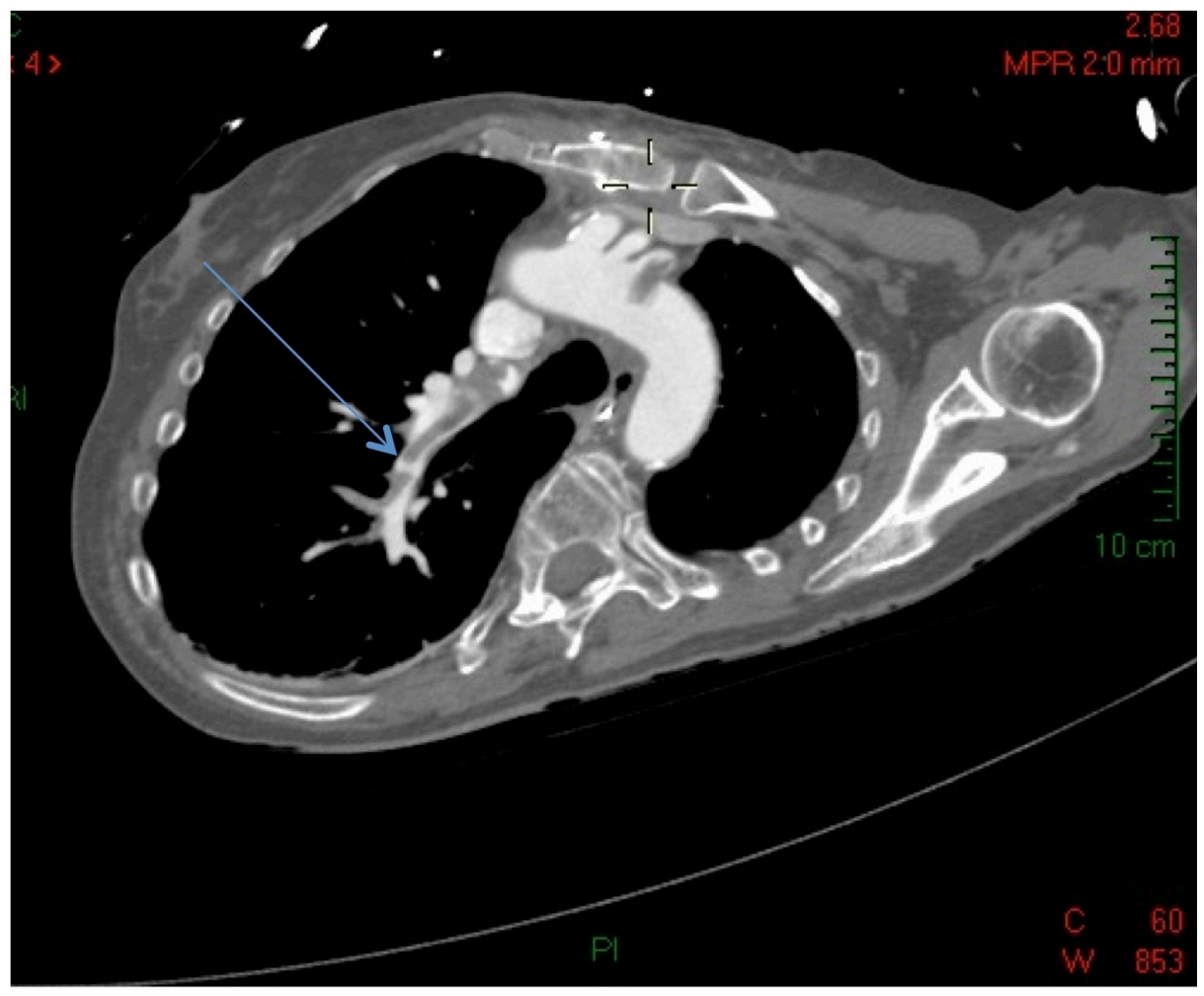

Figure 3: Thrombosis in the right pulmonary artery branches. 
An emergent thrombectomy operation of the arterial thrombosis was performed, followed by prolonged heparin treatment for the pulmonary embolism. After the operation, compression ultrasonography was performed on both legs, demonstrating bilateral deep venous thrombosis.

She underwent successful extubation and was later discharged from the hospital to a rehabilitation centre. The patient was followed up for 8 months after the event, and she is doing fine.

An ambulatory workup for a coagulation profile was performed and did not demonstrate any abnormalities.

It is important to emphasize that on a CTA of the chest performed 3 weeks prior to this event, there was no evidence of thrombi in the pulmonary or the arterial systems.

\section{Discussion}

This is a rare presentation of acute and combined arterial and venous thrombosis, managed in the emergency department by a combined team of emergency physicians, cardiac surgeons and cardiologists, utilizing several imaging modalities, with a good outcome for the patient.

It is important to understand the coexistence of two different acute thrombotic processes in the same patient. The concomitant occurrence of venous and arterial thrombosis is rare, and information in the literature exists only from case reports. Thus, no guidelines exist for diagnosis and management.

When it does occur ${ }^{1-4}$, according to the reports reviewed, the presentation tends to be dramatic, and management must be quick and involve various modalities of imaging and intensive medical and surgical treatment (anticoagulation and surgical/endovascular interventions) ${ }^{1-4}$.

Pulmonary embolism is a known complication of cardiac operations, and other major operations, but we found no descriptions of its coexistence with acute arterial thrombosis, except in the presence of patent foramen ovale [1], thrombi in the right cardiac ventricle and other arteriovenous connections [2] - the so-called "paradoxical embolism", which may be arterial (cerebrovascular stroke, coronary stroke) or venous (pulmonary embolism) ${ }^{1-2}$.

No anatomical connection was found between the arterial and venous systems in our patient on imaging studies or during surgery.

The coexistence of arterial and venous thrombosis is described in hypercoagulable states malignancies, protein $\mathrm{C}$ deficiency, hyperhomocysteinaemia and vasculitis ${ }^{3,4}$, but, according to the literature, even in these cases it is a rare coexistence and usually venous and arterial thrombosis do not occur acutely together.

In some of the reports in the literature, the venous thrombosis is presumed to be an incidental finding, with no acute clinical significance.

The patient in our case underwent screening for concomitant conditions (occult malignancy, hypercoagulation) during her stay in the hospital and on an outpatient basis, and none were found. 
Another point to emphasize is the major development in diagnostic and therapeutic options for vascular thromboembolism during the last decade. At the beginning of the decade most diagnoses were made by angiographic studies, whereas today most are made by noninvasive imaging studies. Also, in many cases where traditionally surgery was used, angiography now serves as a therapeutic modality ${ }^{2,5}$.

\section{Conclusions}

This is a rare case of combined acute venous and arterial thrombosis in a patient with no risk factors except post-cardiac surgery and with no arteriovenous connections.

It was diagnosed in the emergency department using several imaging modalities and treated surgically and medically with good clinical outcomes.

We believe physicians should be aware of the possibility of combined thrombosis and should be acquainted with the available diagnostic and therapeutic tools, which have become more available and numerous in the last several years.

More studies and guidelines for diagnosis and treatment are needed.

\section{Learning Points}

- Physicians should be aware of the possibility of combined arterial and venous thrombosis.

- The situation should be evaluated and treated promptly.

- Evaluation and treatment modalities have greatly evolved during the last decade.

\section{References}

1. Smith JG, Koul S, Roijer A, Holmqvist J, Keussen I, Cwikiel W et al. Acute right ventricular failure caused by concomitant coronary and pulmonary embolism: successful treatment with endovascular coronary and pulmonary thrombectomy, Eur Heart J Acute Cardiovasc Care 2013;2:131-136.

2. Na SJ, Koh YS, Kim TH, Park SC, Shin WS, Chun HJ et al. lliocaval fistula presenting with paradoxical pulmonary embolism combined with high output heart failure successfully treated by endovascular stent graft repair: case report, J Korean Med Sci 2014;29:296-300.

3. Hudzik B, Szkodinski J, Polonski L. Pulmonary embolism and intra-aortic thrombosis in essential thrombocythemia, Br J Haematol 2012;158:562.

4. D'Amato N, Correale M, D'Agostino C. Aortic thrombus and acute pulmonary embolism in an individual heterozygous for the MTHFR C677T mutation, Rev Esp Cardiol ;63:1366.

5. Baumert B, Korner M, Sadeghi Azandaryani M, Rummeny C, Reiser M, Linsenmaier, U. Multidetector computed tomography in the diagnosis of non-traumatic vascular emergencies, Radiologie 2009;49:481-491. 